\title{
Numerical simulations of the mesostructure formation in material extrusion additive manufacturing
}

Serdeczny, Marcin P.; Comminal, Raphaël; Pedersen, David B.; Spangenberg, Jon

Published in:

Additive Manufacturing

Link to article, DOI:

10.1016/j.addma.2019.05.024

Publication date:

2019

Document Version

Peer reviewed version

Link back to DTU Orbit

Citation (APA):

Serdeczny, M. P., Comminal, R., Pedersen, D. B., \& Spangenberg, J. (2019). Numerical simulations of the mesostructure formation in material extrusion additive manufacturing. Additive Manufacturing, 28, 419-429. https://doi.org/10.1016/j.addma.2019.05.024

\section{General rights}

Copyright and moral rights for the publications made accessible in the public portal are retained by the authors and/or other copyright owners and it is a condition of accessing publications that users recognise and abide by the legal requirements associated with these rights.

- Users may download and print one copy of any publication from the public portal for the purpose of private study or research.

- You may not further distribute the material or use it for any profit-making activity or commercial gain

- You may freely distribute the URL identifying the publication in the public portal 
Keywords: material extrusion additive manufacturing; fused deposition modeling; numerical simulations; mesostructure; porosity.

\section{Abstract}

A computational fluid dynamics model is used to predict the mesostructure formed by the successive deposition of parallel strands in material extrusion additive manufacturing. The numerical model simulates the extrusion of the material onto the substrate. The model takes into account the effect of the presence of the previously extruded material on the shape of the subsequently deposited strands. The simulated mesostructures are compared to optical micrographs of the mesostructures of 3D-printed samples, and the predictions agree well with the experiments. In addition, the influence of the layer thickness, the strand-to-strand distance, and the deposition configuration (with aligned or skewed layers) on the formation of the mesostructure is investigated. The simulations provide detailed information about the porosity, the inter- and intra-layer bond line densities, and the surface roughness of the mesostructures, which potentially can be used in a model-based slicing software.

\section{Introduction}

Material extrusion Additive Manufacturing (AM) [1] (also known as fused filament fabrication and fused deposition modeling) is one of the most popular 3D printing techniques [2,3]. During fabrication, a strand of material is deposited along a predefined toolpath, which builds the object layer by layer. This process has been widely used for rapid prototyping and tooling, and its potential applications start reaching fields, such as the aerospace industry [4], biomedical sciences [5] and civil engineering [6], where highly customable products with complex geometries are desirable. Material extrusion AM technologies have already been developed for a wide range of materials, including fiber-reinforced thermoplastics [7], ceramic pastes [8,9], concrete [6,10], and hydrogel suspensions of living cells [11,12].

The parts manufactured with material extrusion AM have usually lower mechanical properties than with conventional polymer processing [13,14], because of the presence of pores in the internal mesostructure formed by the deposited strands [15-17]. The bonding areas between the strands are the weak points of the mesostructure, where a mechanical failure is likely to occur [13,18]. The porosity and the bonding areas depend on the layer thickness, the strand-to-strand distance and the printing speed, as those parameters influence the cross-sectional shape of the extruded stands [19-24]. Furthermore, the mesostructure of the fabricated component is typically anisotropic, which causes the dependency of the mechanical strength on the build orientation and the tool path [13,25-28]. In addition, the layered deposition of material determines the surface finish and the build time of the part [29-32]. For instance, a small layer thickness improves the surface roughness, but leads to a higher build time [13,28]. Thus, many experimental studies have focused on finding optimal combinations of the process parameters [33-35].

In complement to experimental studies, numerical simulations can deliver a vast amount of information about the process and have proven to be a predictive tool in the analysis of different manufacturing methods [36-39]. In the context of material extrusion AM, the extrusion flow has been investigated using two-dimensional [40-42], and three-dimensional Computational Fluid Dynamics (CFD) simulations [43-48]. Du et al. [43] and Xia et al. [45] have simulated the deposition of successive strands on top of each other, to predict their cooling time and the inter-layer contact area. Comminal et al. [46] have simulated the deposition of a single strand and investigated the dependency of its cross-sectional shape on the layer thickness and the printing speed. The CFD model was subsequently validated by experimental measurements in [49]. In addition, the work in [49] has also shown that there are discrepancies between the measured cross-sections and the analytical approximations that are used in slicer algorithms. The CFD model of Comminal at al. [46] was also used to investigate the influence of the shape of the extrusion nozzle on the strand crosssection [50], as well as the impact of the printing head acceleration profiles on the shape of the strand at corners [51]. The microarchitecture of porous scaffolds was simulated by Gleadall et al. [24], using a three-dimensional voxelised volume conservation model. To the authors' knowledge, the mesostructure formation in material extrusion AM has never been simulated with CFD models.

This paper presents a methodology to simulate the deposition of multiple layers of parallel strands forming a representative volume element of the mesostructure. Toolpaths with parallel strand deposition are typically used for contouring the shell of the components, and for printing the horizontal surfaces. The simulations of the strand deposition take into account the influence of the 
presence of the previously deposited material on the morphology of subsequently extruded strands. The mesostructures predicted by the numerical simulations are used to evaluate the porosity, the inter- and intra-layer bond line densities, and the surface roughness of the representative volume element. The influence of the layer thickness and the intra-layer strand-to-strand distance is investigated for two deposition configurations, with aligned and skewed layers. The remaining structure of this paper is as follows. The computational model and the numerical methods of the simulations are presented in Section 2. The simulated mesostructures for the different processing parameters are shown and discussed in Section 3. Section 4 summarizes the conclusions of the study.

\section{Methods}

\subsection{Computational model}

The extrusion and deposition of the strands was simulated with a CFD model. Figure 1 (left) shows the geometry of the numerical model when the deposition of the first strand was simulated. The molten material entered the cylindrical nozzle with an average velocity $U$. The nozzle had a diameter $D$ and was placed above a substrate at a distance $t$, corresponding to the layer thickness. For the deposition of the first strand, the substrate was a flat surface representing the build plate of a 3D printer. The substrate was moving with respect to the nozzle with a constant velocity $V$, which was the printing speed. The material that was extruded onto the moving substrate was conveyed towards the outlet boundary of the calculation domain.

The deposition of the subsequent strands was simulated sequentially. For the deposition of each next strand, the previously extruded strands were now part of the substrate, see Figure 1 (right). At the end of each simulation, the cross-section of the latest deposited strand was measured at the outlet boundary. The strand cross-section was then extruded through the entire computational domain, to create a solid body that was merged with the previous substrate. Finally, the geometry of the new substrate was translated horizontally (for the deposition of an adjacent strand) or vertically (for the deposition of a new layer), according to the deposition planning of the strands.
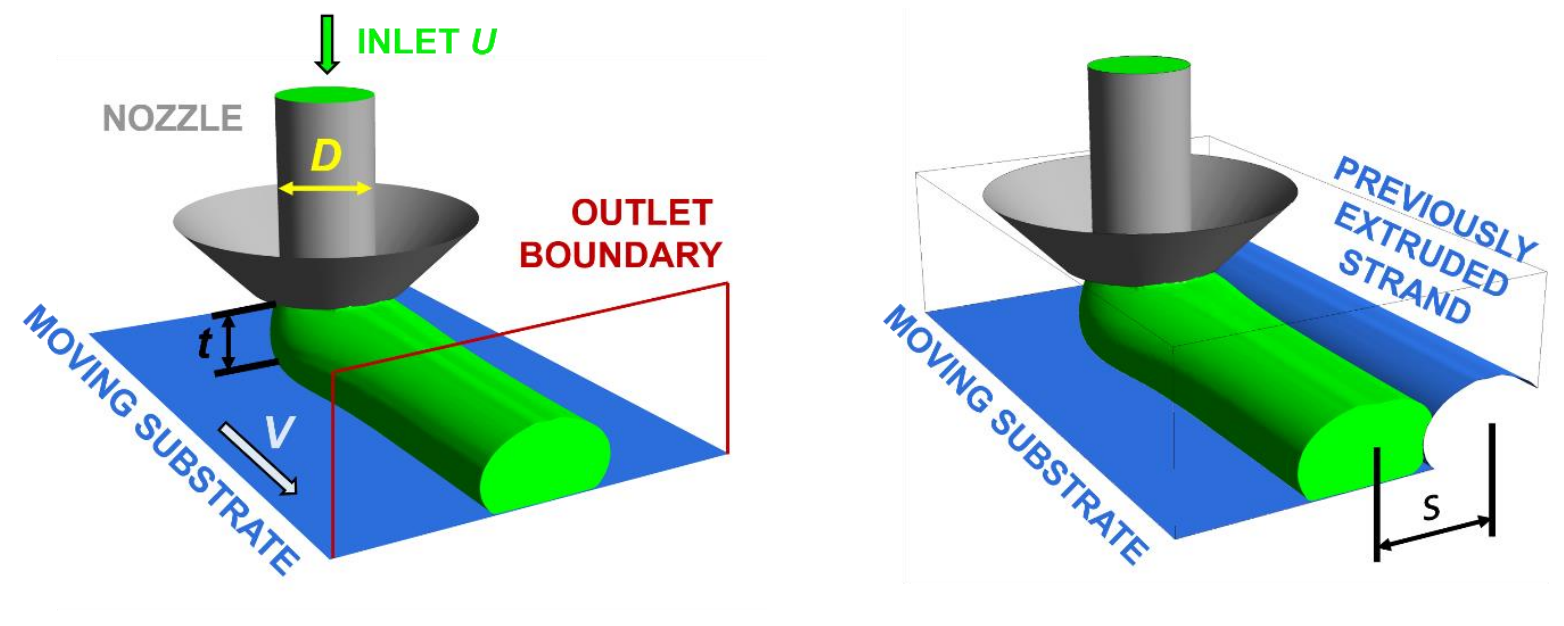

Figure 1. Geometry of the computational domain. Simulation of the first strand deposition (left); and the subsequent strand deposition (right).

Two deposition configurations were considered: the aligned and skewed cases; see Figure 2. In both cases, each layer consisted of a regular array of strands, periodically deposited with the strand-to-strand distance $s$. In the aligned case, the layers were laid on top of each other with the same pattern. For the skewed case, the layers were staggered with an interval of $s / 2$. In total, the deposition of 4 layers containing 4 strands each was simulated. The deposition order of the 16 strands is indicated in Figure 2 . In the skewed case, three additional strands (colored in grey in Figure 2) were placed onto the substrate, in order to support the subsequent layers. The deposition of those additional strands was not simulated and their cross-sections were duplicated from the previously laid strands, as indicated with arrows in Figure 2. 

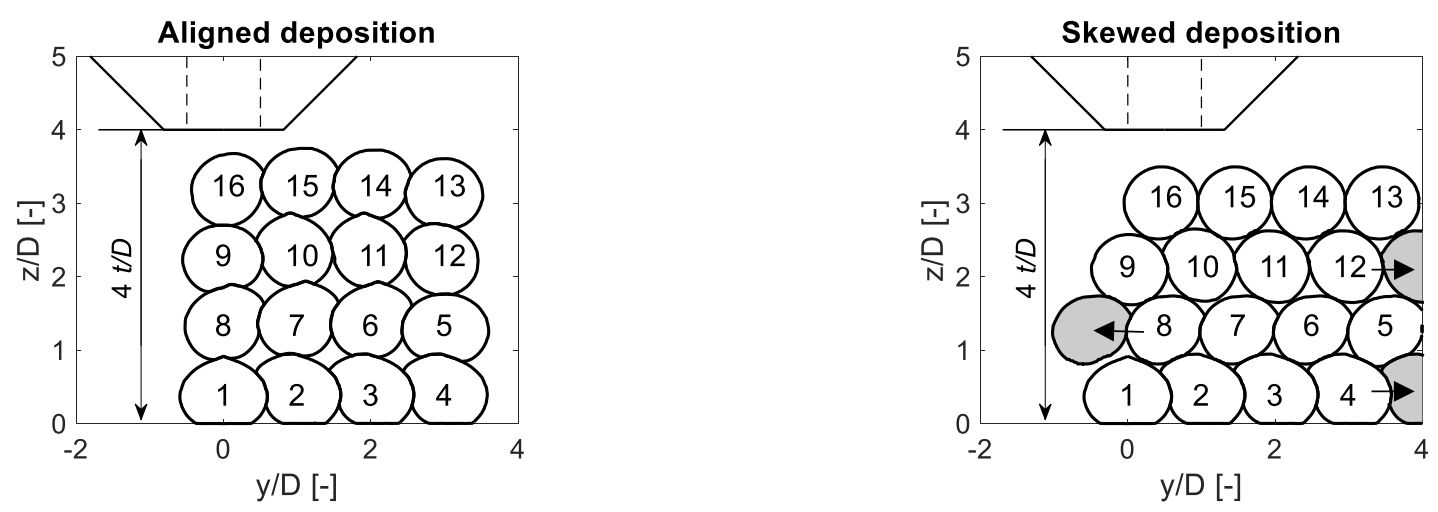

Figure 2. Deposition order of the strands, for the aligned and skewed cases. The strands marked in grey (skewed case) are duplicates of the previously laid strands, as indicated by the arrows. The dashed lines represent the channel of the extrusion nozzle.

The deposition flow is governed by the Navier-Stokes equations, which account for the conservation of mass and momentum. In addition, the following assumptions were made in the model:

- The molten material fully adhered to the substrate, the previously deposited material, and the nozzle walls. No-slip boundary conditions were applied on those solid surfaces.

- The fluid had a constant density. It was assumed that the thermal shrinkage had a secondary effect on the morphology of the formed mesostructure, as compared to the influence of the previously deposited strands on the subsequently extruded material. This assumption is similar to [24].

- The fluid had a constant viscosity. It has been shown in a previous study [52] that the variations in the dynamic viscosity of the fluid have a negligible influence on the strand morphology, as long as the flow is in the creeping flow regime (i.e. the Reynolds number $R e$ is below 1), which is typically the case in material extrusion AM.

- The extruded material solidified after the deposition, but the model did not solve for the energy equation and the solidification. The material from the previously extruded strands was included as a rigid solid during the simulations of the subsequent strands deposition. It was assumed that the reheating of the previously deposited strands by the newly extruded material is a local effect that does not influence their shapes, as polymers typically have a low thermal conductivity. On the other hand, the presence of the solidified strands influences the deposition of subsequently extruded material, which affects the formation of the mesostructure. This assumption is similar to [24].

The printing parameters, and the material properties of the numerical model are summarized in Table 1. The values of the layer thickness were chosen identical to those of our previous numerical and experimental studies [49]. The strand-to-strand distance was varied to investigate the influence of the presence of solidified material on the shape of subsequently deposited strands and the formation of the mesostructure. The values of the density and viscosity of the simulated fluid are also listed in the table. However, their actual values have a negligible influence on the results, as long as the flow remains in the creeping regime $(\operatorname{Re}<1)$. Thus, the deposition flow was entirely parametrized by three dimensionless characteristic quantities: the normalized layer thickness $t / D$, the normalized strand-to-strand distance $s / D$ and the velocity ratio $V / U$. The normalized layer thickness and strand-to-strand distance parametrize the geometry of the model. The velocity ratio (kept constant within this study) quantifies the amount of extruded material with respect to the printing speed. The numerical results will also be presented in non-dimensional forms, where all lengths are normalized by the nozzle diameter. Therefore, the results are equally valid for different nozzle diameters and printing speeds, as long as the dimensionless quantities $t / D, s / D$ and $V / U$ of the simulated cases are unchanged. 
Table 1.Summary of the simulation parameters used in the different cases.

Dimensional parameters

\begin{tabular}{|c|c|c|c|c|c|c|c|c|}
\hline Variable & Symbol & Unit & Case 1 & Case 2 & Case 3 & Case 4 & Case 5 & Case 6 \\
\hline Layer thickness & $t$ & $\mathrm{~mm}$ & \multicolumn{2}{|c|}{0.24} & \multicolumn{2}{|c|}{0.32} & \multicolumn{2}{|c|}{0.4} \\
\hline Strand-to-strand distance & $s$ & $\mathrm{~mm}$ & 0.56 & 0.58 & 0.40 & 0.48 & 0.4 & 0.46 \\
\hline Deposition configuration & - & - & \multicolumn{6}{|c|}{ Aligned / Skewed } \\
\hline Nozzle diameter & $D$ & $\mathrm{~mm}$ & \multicolumn{6}{|c|}{0.4} \\
\hline Average velocity inside the nozzle & $U$ & $\mathrm{~mm} / \mathrm{s}$ & \multicolumn{6}{|c|}{20} \\
\hline Printing head velocity & $V$ & $\mathrm{~mm} / \mathrm{s}$ & \multicolumn{6}{|c|}{20} \\
\hline Density & $\rho$ & $\mathrm{kg} / \mathrm{m}^{3}$ & \multicolumn{6}{|c|}{1000} \\
\hline Dynamic viscosity & $\eta$ & $\mathrm{Pa} \cdot \mathrm{s}$ & \multicolumn{6}{|c|}{1000} \\
\hline
\end{tabular}

Non-dimensional parameters

\begin{tabular}{|c|c|c|c|c|c|c|c|c|}
\hline Variable & Symbol & Unit & Case 1 & Case 2 & Case 3 & Case 4 & Case 5 & Case 6 \\
\hline Normalized layer thickness & $t / D$ & - & \multicolumn{2}{|c|}{0.60} & \multicolumn{2}{|c|}{0.80} & \multicolumn{2}{|c|}{1.00} \\
\hline Normalized strand-to-strand distance & $s / D$ & - & 1.40 & 1.45 & 1.00 & 1.20 & 1.0 & 1.15 \\
\hline Velocity ratio & $V / U$ & - & \multicolumn{6}{|c|}{1.0} \\
\hline Reynolds number & $\begin{array}{c}R e= \\
\rho U D / \eta\end{array}$ & - & \multicolumn{6}{|c|}{$\sim 10^{-5}$} \\
\hline
\end{tabular}

\subsection{Principles of the numerical simulations}

The flows were simulated with ANSYS Fluent R18.2. The computational domains were discretized with a structured Cartesian cutcell mesh, and the governing equations of the flow were discretized with a collocated finite volume method. An example of the mesh used in one of the simulations is represented in Figure 3. The maximum size of the control volumes was globally set to 20 $\mu \mathrm{m}$ and refined to $10 \mu \mathrm{m}$, in the region where the strand was deposited. The transient flows were solved with the implicit coupled pressure-velocity scheme. The free-surface flow was simulated as a two-phase flow of the molten plastic and the surrounding air, and their interface (i.e. the free-surface of the molten plastic) was tracked with the coupled level-set / volume-of-fluid method [5355]. The deposition flows reached steady states in about $0.6 \mathrm{~s}$, which is when the numerical results were registered. More details about the numerical method can be found in our previous publication [46].
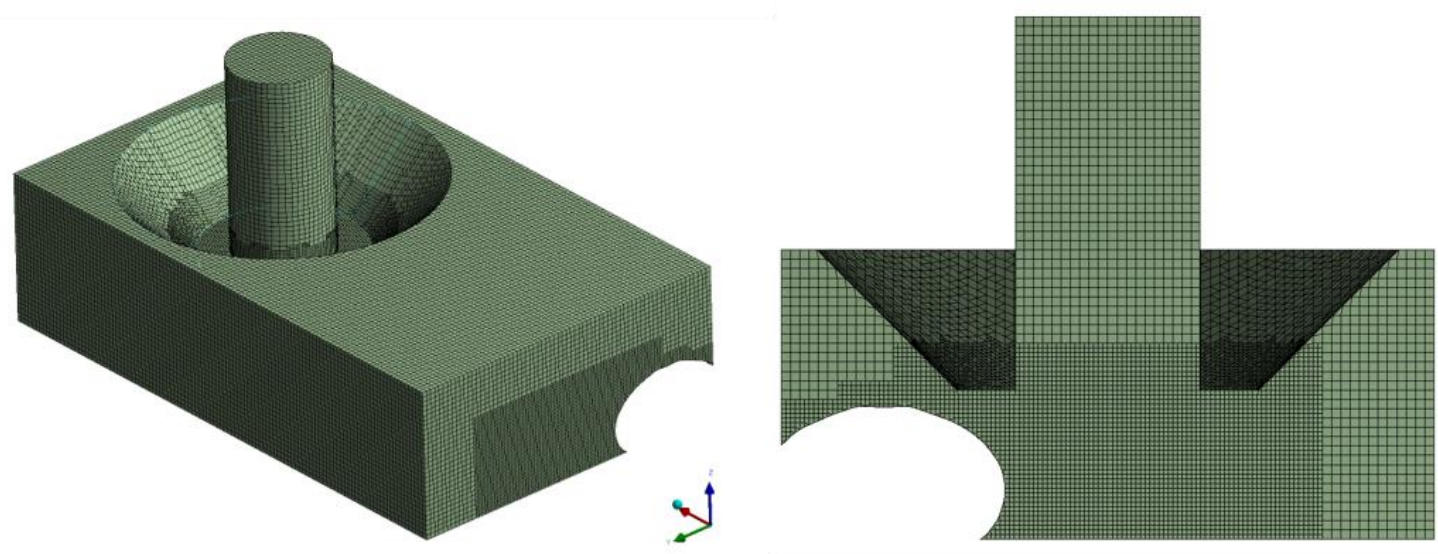

\section{Figure 3. Example of Cartesian cut-cell mesh used in one of the simulations: isometric perspective view (left) and cut view} through a vertical middle plane of the nozzle (right).

\subsection{Post-processing calculations}

After the simulations, the contours of the strands were extracted from the numerical results and analyzed in Matlab. The mesostructures were obtained by combining the contours of all the deposited strands. Examples of the resulting mesostructures are shown in Figure 4, for the aligned and skewed deposition configurations. The porosity, the surface roughness, and the bond line densities were calculated as follows: 
- The porosity corresponds to the area fraction of the pores inside the representative volume element delimited by the black dashed lines in Figure 4. In the cases of the aligned deposition configuration, the representative volume element was a rectangle, while it was a skewed box, in the cases of the skewed deposition configuration; see Figure 4 . The vertices delimiting the edges of the representative volume elements were located at the centers of the strands that were deposited at the extremity of the mesostructures. In this way, edge effects were avoided in the calculations of the porosity.

- The surface roughness of the horizontal and vertical edges of the mesostructures were evaluated with the arithmetic mean deviation $R_{a}$ with respect to the mean line of the profile. The mean line for each profile was calculated such that the sum of areas enclosed by the profile above the mean line was equal to the sum of those below the mean line. The horizontal and vertical profiles used for the calculation of the surface roughness are respectively highlighted in blue and red in Figure 4. Note, that the surface roughness was only estimated for the aligned deposition, as the skewed configuration is usually applied for the infill of the part. The surface roughness was calculated as:

$$
R_{a}=\frac{1}{n} \sum_{i=1}^{n}\left|y_{i}\right|
$$

where $y_{i}$ is the distance of the sampling points from the mean line, and $n$ is the total number of sampling points. The sampling points were equally spaced along the profiles, by piecewise polynomial interpolations.

- The bond line densities were calculated separately for the horizontal (inter-layer) and vertical (intra-layer) surfaces. The inter-layer (or intra-layer) bond line density $\rho_{H}$ (or $\rho_{V}$ ) corresponds to the sum of the lengths of the bond lines projected on the horizontal (or vertical) plane, divided by the width $W$ (or height $H$ ) of the representative volume element and the integer $N_{L^{-}}\left(\right.$or $\left.N_{A^{-}}-1\right)$ :

$$
\rho_{H}=\frac{1}{N_{L}-1} \sum_{k=1}^{M} \frac{P_{H}\left(l_{k}\right)}{W}
$$

$$
\rho_{V}=\frac{1}{N_{A}-1} \sum_{k=1}^{M} \frac{P_{V}\left(l_{k}\right)}{H}
$$

where $P_{H}\left(l_{k}\right)$ and $P_{V}\left(l_{k}\right)$ are the lengths of the $k^{\text {th }}$ bond line $l_{k}$ projected on the horizontal and vertical plane, respectively. $N_{L}$ is the number of layers and $N_{A}$ is the number of adjacent strands per layer, excluding the duplicated one. $M$ is the total number of bond lines inside the representative volume element. When a bond line intersected the boundary of the representative volume element, the horizontal and vertical projections only took into account the sections of the bond lines that were inside the representative volume element. The horizontal and vertical projections of the bond lines are illustrated in Figure 5.
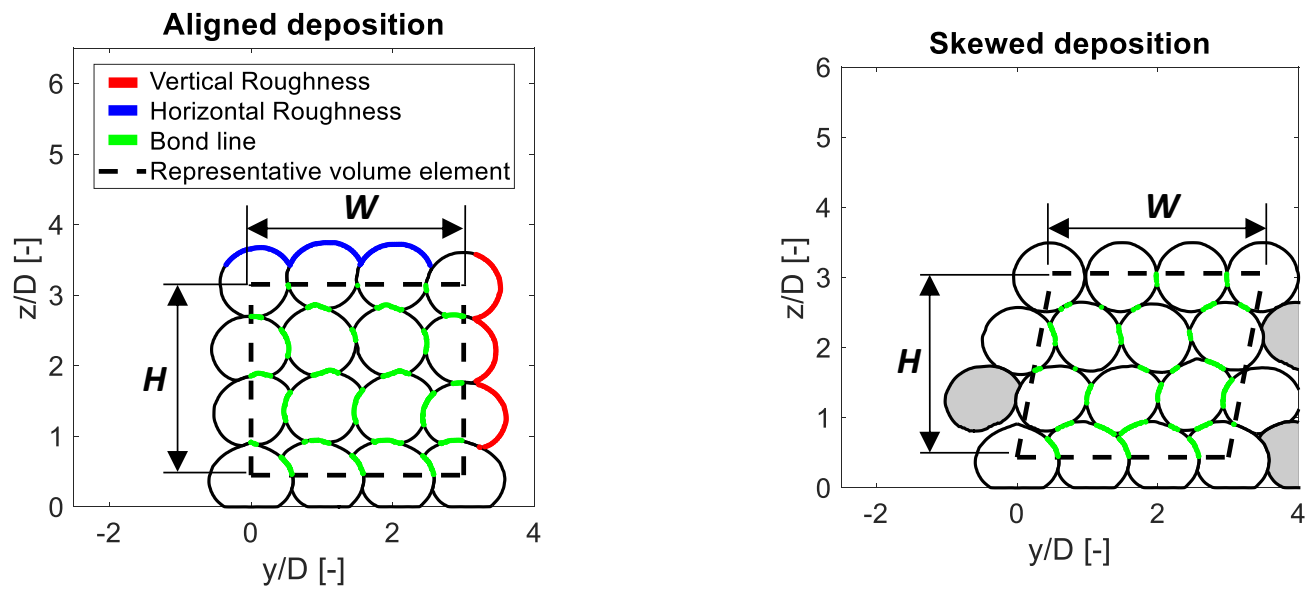

Figure 4. Examples of mesostructure used in the post-processing calculations. $W$ and $H$ are the width and height of the representative volume element, respectively. 


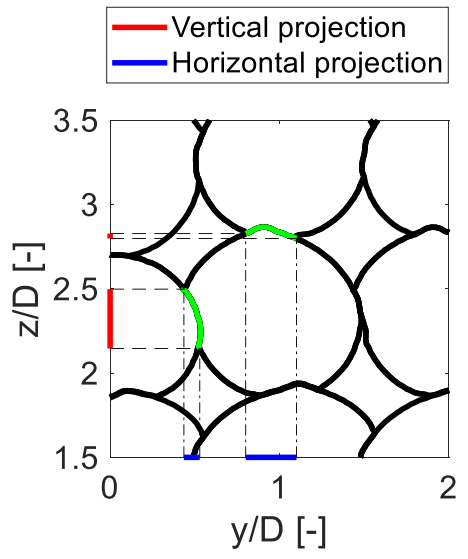

Figure 5. Illustration of the vertical and horizontal projections of the bond lines.

\section{Results and discussions \\ 3.1. Comparison with experimental measurements}

The mesostructures predicted by the numerical simulations were compared with the cross-sections of parts fabricated with material extrusion AM. Four PLA specimens were manufactured with a desktop 3D printer (BQ Hephestos 2). The processing parameters were selected such as to obtain mesostructures for which the contour of the strands were distinguishable and the formation of voids was observable. After the fabrication, the samples were cut through a plane orthogonal to the printing direction. The cross sections were polished and examined under an optical microscope. The comparison of the simulated mesostructures with the experimental observations is presented in Figure 6. The predicted mesostructures agree qualitatively well with those observed in the experiments. For the aligned configuration, the pores have a diamond shape. For the skewed configuration, the shape of the pores depends on the strand-to-strand distance. When the strand-to-strand distance is larger than the strand width, the pores have an hourglass shape. On the contrary, when the strand-to-strand distance is smaller than the strand width, the hourglass shape of the pore is broken into two triangular pores.

The micrographs were also analyzed quantitatively, and the comparison of the measured and simulated porosities, and bond line densities is reported in Table 2. The porosities were determined through image analysis, based on the difference of pixels' color between the strands and voids. It can be seen that the measured values compare well with the simulation results. This shows that the assumptions undertaken when solving the flow equations are reasonable for the prediction of the porosity. The bond line densities were measured manually from the micrographs. In most of the cases, the agreement between the measured bond line densities and the numerical results is good. However, for some particular cases $(t / D=1.0, s / D=1.0$ and $t / D=0.8, s / D=1.2$ with the aligned deposition configuration) discrepancies occurred. In those situations, the bond line densities are very sensitive to the strands' cross-sectional area, and a slight increase in the strands' area can lead to the formation of a bond line (with a positive length), instead of a punctual contact (with zero length). Therefore, in those specific cases, an improved agreement with the experiments might have been obtained by including thermal shrinkage of the material into the simulations. Even so, overall the comparison with experimental measurements showed that the model is capable of predicting the influence of the printing parameters on the mesostructure formation in material extrusion AM. 

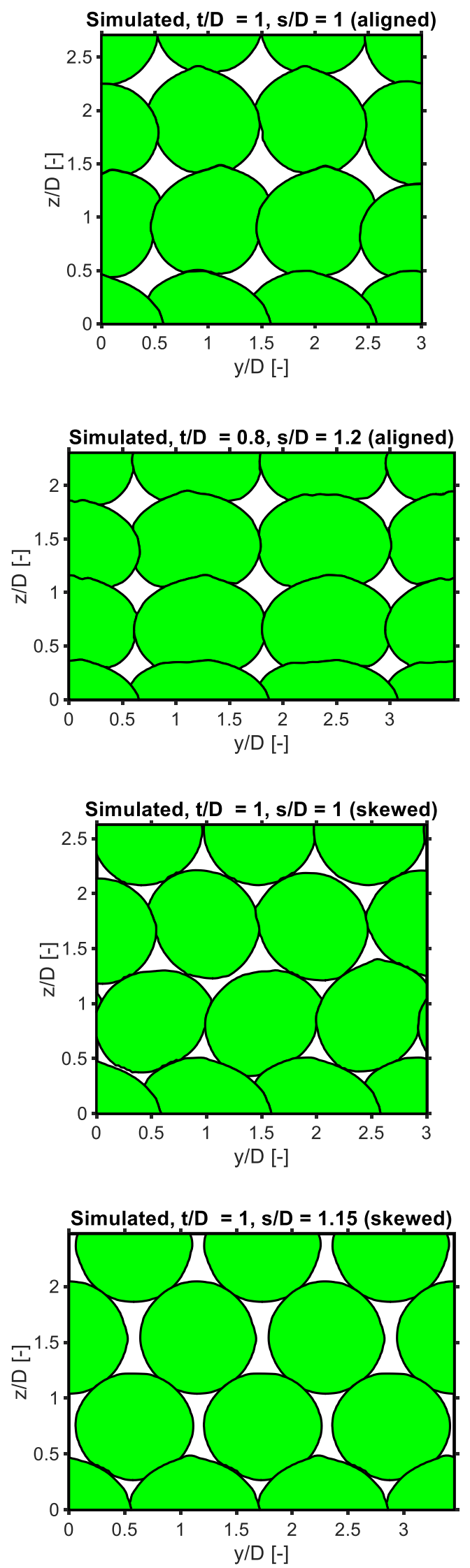
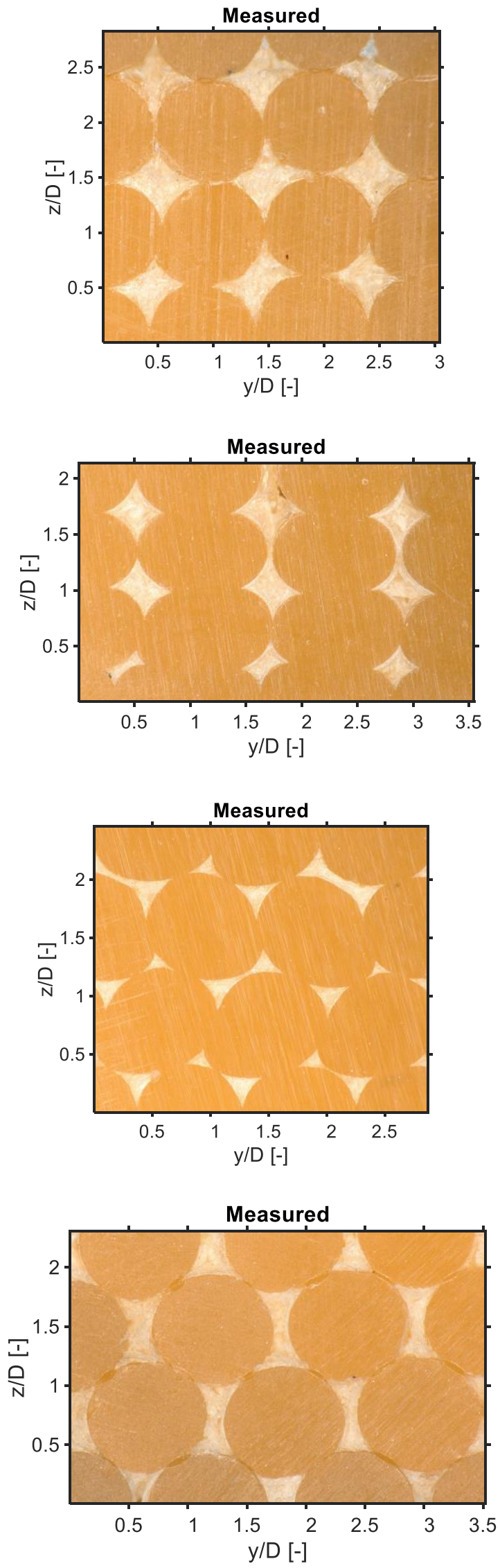

Figure 6. Qualitative comparison of the simulated (left) and measured (right) mesostructures for different printing parameters. 
Table 2. Quantitative comparison of porosity and bond line densities between simulated and measured mesostructures.

\begin{tabular}{|c|c|c|c|c|c|c|c|c|}
\hline \multirow{2}{*}{ t/D [-] } & \multirow{2}{*}{ S/D [-] } & \multirow{2}{*}{ Configuration } & \multicolumn{2}{|c|}{ Porosity [-] } & \multicolumn{4}{|c|}{ Bond Line Density [-] } \\
\cline { 4 - 9 } & & & Simulated & Measured & Simulated & Measured & Simulated & Measured \\
\hline 1.0 & 1.0 & Aligned & 0.14 & $0.15 \pm 0.03$ & 0.44 & $0.30 \pm 0.01$ & 0.38 & $0.22 \pm 0.01$ \\
\hline 0.8 & 1.2 & Aligned & 0.10 & $0.10 \pm 0.02$ & 0.60 & $0.58 \pm 0.02$ & 0.44 & $0.26 \pm 0.01$ \\
\hline 1.0 & 1.0 & Skewed & 0.08 & $0.06 \pm 0.01$ & 0.32 & $0.37 \pm 0.01$ & 0.45 & $0.50 \pm 0.01$ \\
\hline 1.0 & 1.15 & Skewed & 0.13 & $0.11 \pm 0.03$ & 0.29 & $0.30 \pm 0.01$ & 0.26 & $0.31 \pm 0.01$ \\
\hline
\end{tabular}
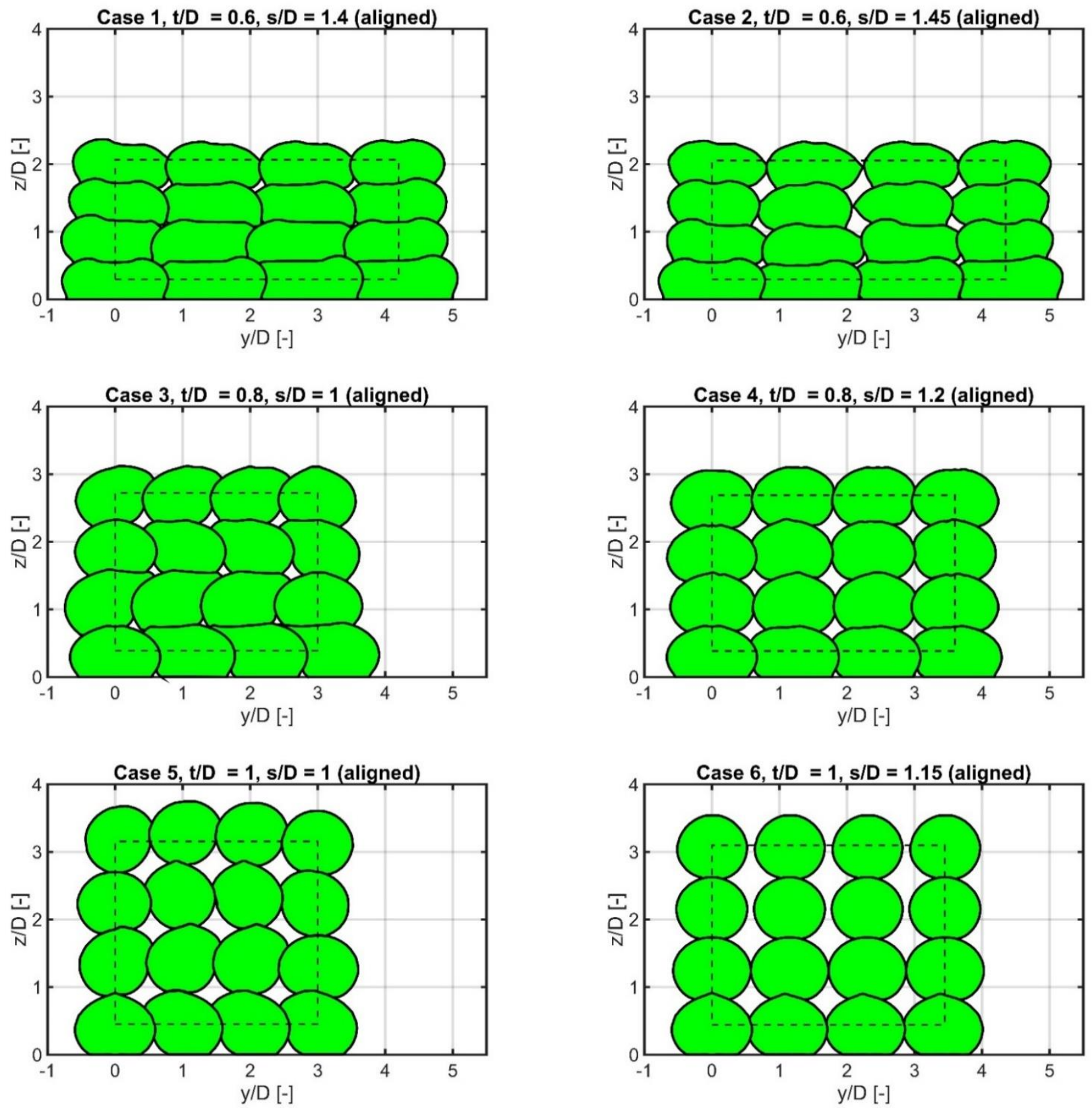

Figure 7. Simulated mesostructures for the aligned deposition configuration. The representative volume element for the calculation of the porosity and the bond line density is represented by the black dashed lines. 


\subsection{Influence of the printing parameters on the mesostructure} 3.2.1. Strand morphology

The cross-sections of all the simulated mesostructures with the aligned deposition configuration are shown in Figure 7. In the cases 2, 4 and 6 of the aligned deposition configuration, the strand-to-strand distance is close to or larger than the strand width. This leads to little or no contact between the printed strands within the same layer. It can be seen that decreasing the layer thickness flattens the strands, by virtue of the volume conservation (constant density assumption), while the pore size decreases. In the cases 1, 3 and 5 of the aligned deposition configuration, the neighboring strands are more compact and have larger intra-layer bonds, as the values of the strand-to-strand distances were smaller than the width of the strands. In these cases, the presence of the previously extruded material has an influence on the morphology of the subsequently deposited strands. Thus, the mesostructure is affected by the order of deposition of the strands.
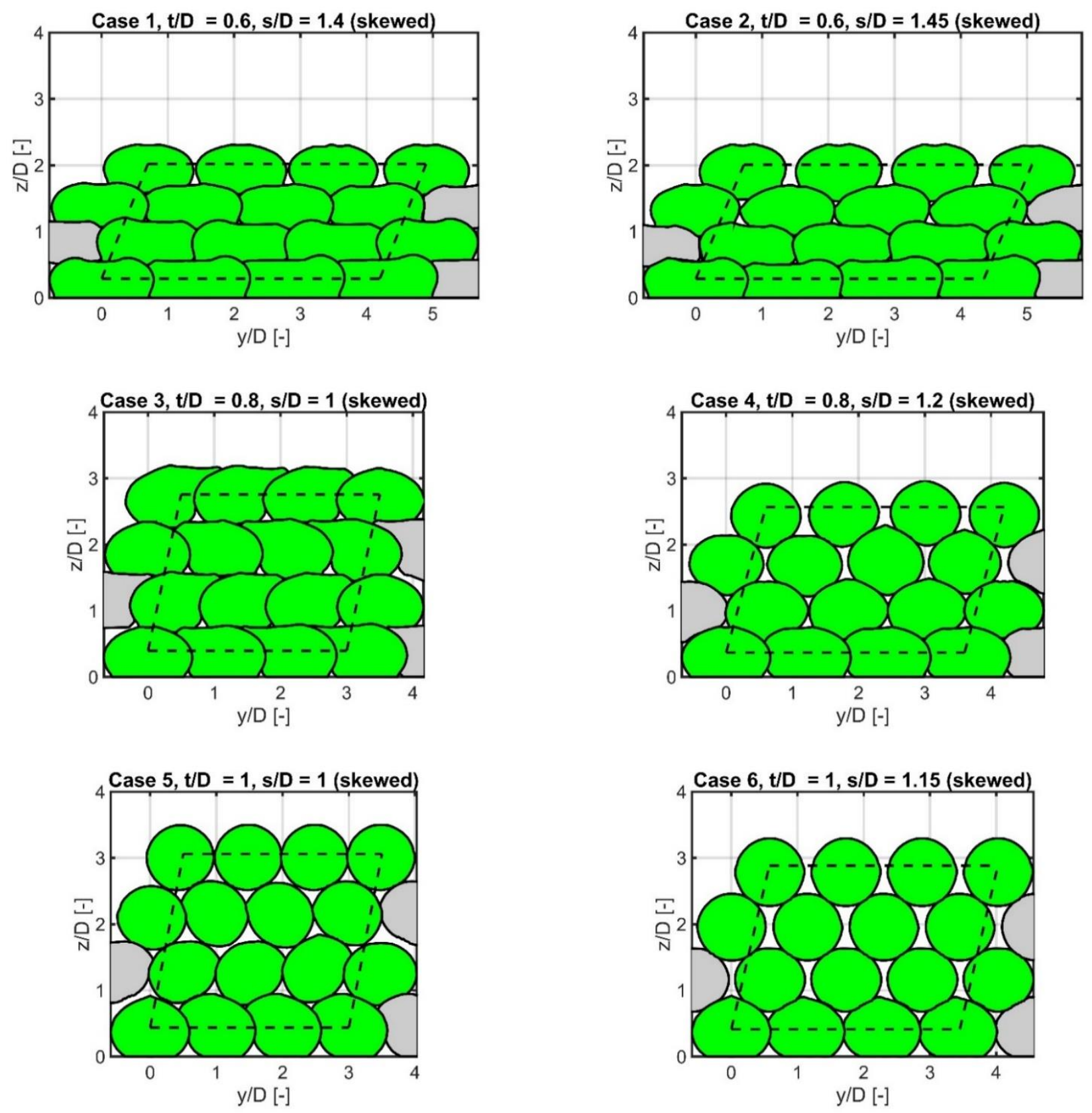

Figure 8. Simulated mesostructures for the skewed deposition configuration. The representative volume element for the calculation of the porosity and the bond line density is represented by the black dashed lines. The grey color indicates the duplicated strand contours. 
1 Figure 8 represents all the simulated mesostructures with the skewed deposition configuration. The shapes of the strands depend on the layer thickness and the strand-to-strand distance, as for the aligned deposition. The strand cross-sections become wider when the layer thickness is decreased, because of the volume conservation. Moreover, when the strand-to-strand distance is smaller than the strand width, the strand cross-sections are asymmetrical and dependent on the order of deposition. In addition, the strands in the top layers have a more circular shape than the strands in the bottom layers. This occurs because the actual distance between the nozzle orifice and the already deposited material becomes larger and eventually exceeds the specified layer thickness as more layers are deposited, because the skewed strands get accommodated in the gaps of the previous layer. To avoid this effect, the layer thickness would need to be reduced by the difference between the actual gap and layer thickness.

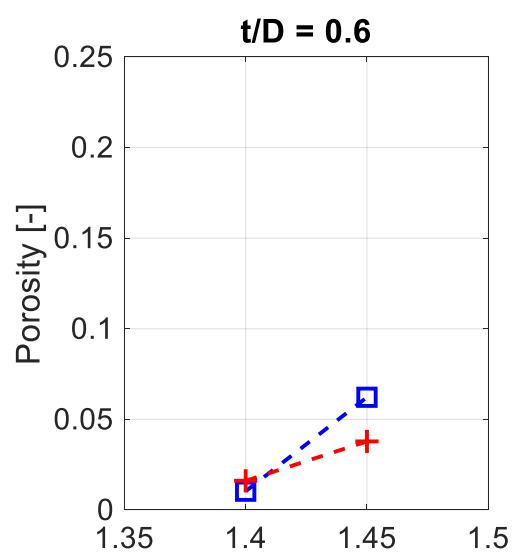

9
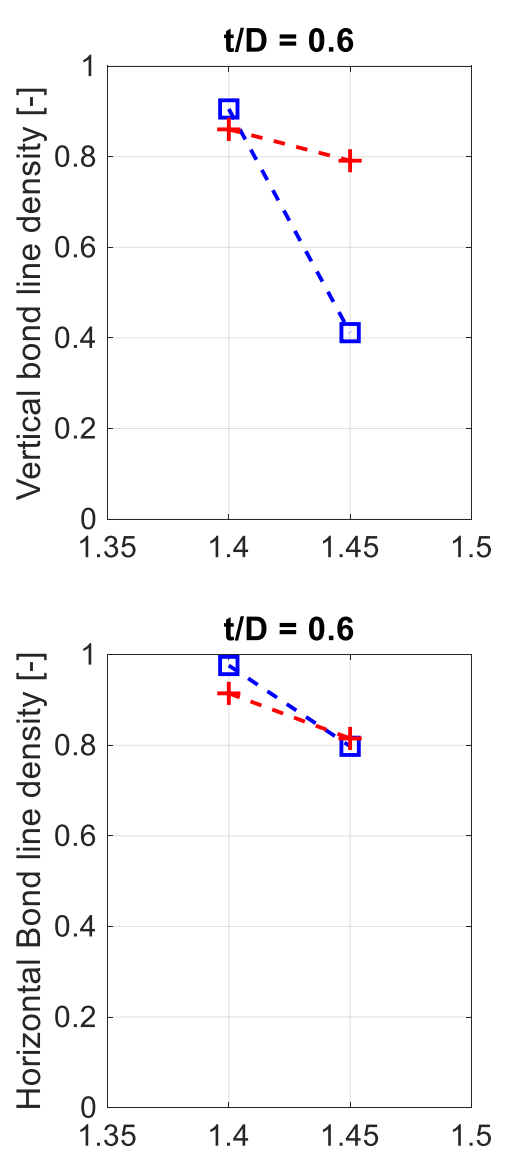

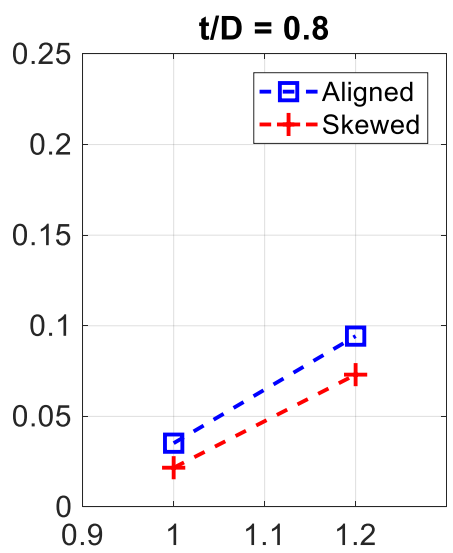

Strand-to-strand distance, s/D

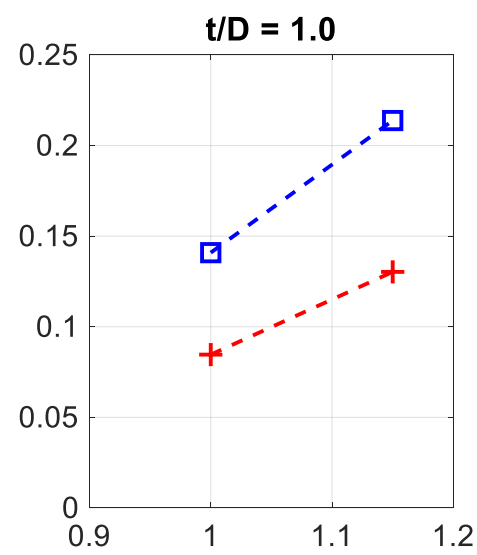

uctures.
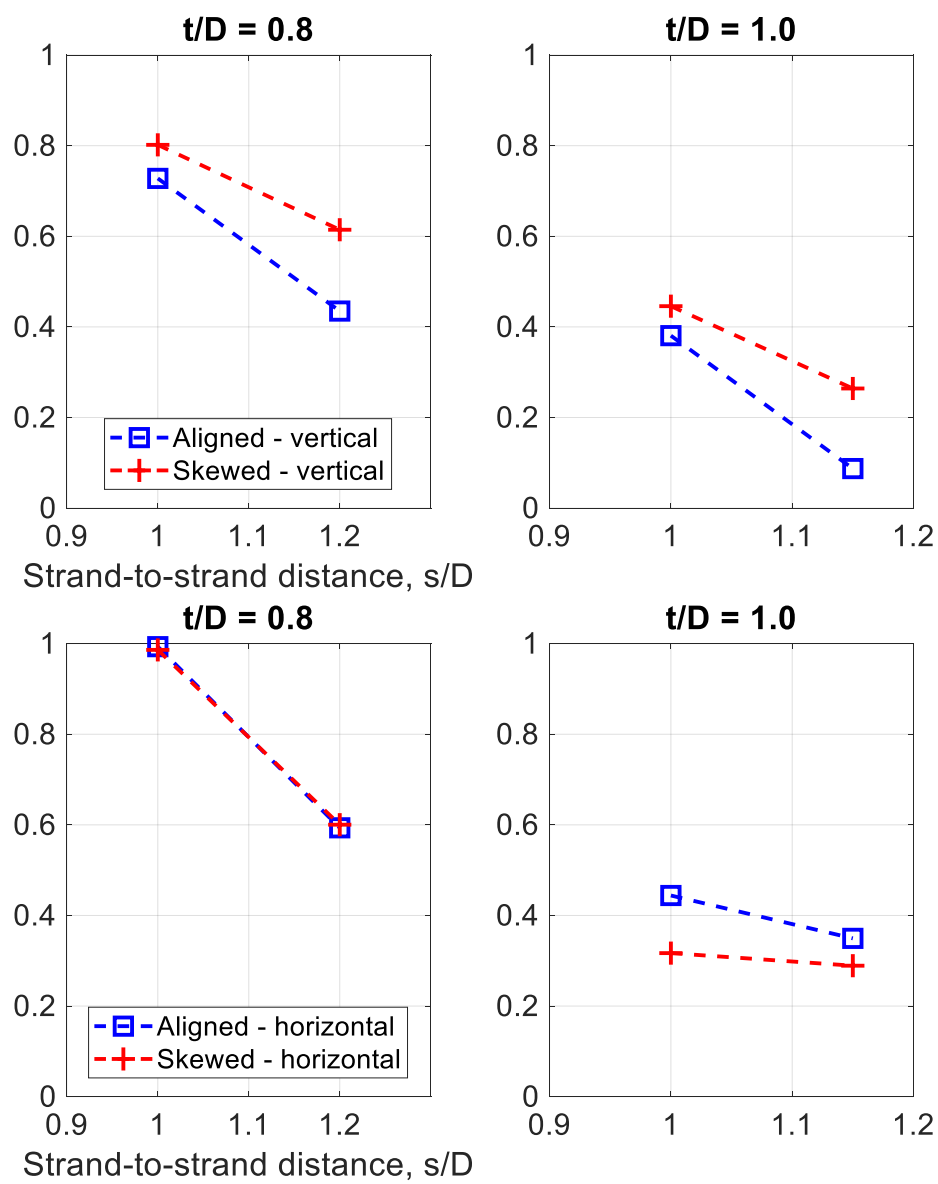

Figure 10. Vertical (intra-layer) and horizontal (inter-layer) bond line densities of the simulated mesostructures. 
The results of the porosity predictions for the different mesostructures are presented in Figure 9. The porosity increases with the strand-to-strand distance and the layer thickness. Moreover, the skewed deposition configuration led to lower porosities than for the aligned configuration, except in Case $1(t / D=0.6, s / D=1.4)$, where both deposition configurations resulted in a very low porosity. Furthermore, the differences of porosity between the skewed and the aligned deposition configurations become more apparent for the larger layer thickness. Within the range of the investigated parameters, Case $1(t / D=0.6, s / D=1.4)$ yielded the lowest porosity, for both of the aligned and the skewed deposition configurations.

\subsubsection{Bond line densities}

The horizontal (inter-layer) and vertical (intra-layer) bond line densities of the different simulated cases are presented in Figure 10. The variations in the bond line densities with respect to the processing parameters $t / D$ and $s / D$ show the inverse trends as for the porosity. The horizontal and vertical bond line densities decrease when the strand-to-strand distance or the layer thickness increases. When the layer thickness is decreased, the flattening of the strand results in a larger surface for the horizontal (inter-layer) bonds. The vertical (intra-layer) bonding is also enhanced when the strand width increases above the strand-to-strands distance, because the extruded material tends to fill the pores. In general, the skewed configuration led to larger intra-layer bond line densities, except in Case 1, where both configurations provided a large intra-layer bond line density. The horizontal bond line density seems independent on the deposition configuration, except in the cases with the large layer thickness $(t / D=1.0)$, where the aligned configuration yielded longer horizontal bond lines.

\subsubsection{Surface roughness}

The vertical and horizontal surfaces of the simulated mesostructures are represented in Figures 11 and 12, respectively. The arithmetic average roughness $R_{a}$ of the profiles are compared in Figure 13. The numerical results show that decreasing the layer thickness improves the surface quality of both the vertical walls and the horizontal surfaces. Smoother vertical walls are obtained by changing the shape of the strands from being circular to oblong, when decreasing the layer thickness. The influence of the layer thickness on the horizontal surface roughness can be seen when comparing Case $3(t / D=0.8, s / D=1.0)$ and Case $5(t / D=1.0, s / D=1.0)$, which have the same strand-to-strand distance, but different values of the layer thickness. In these cases, the lower roughness of the horizontal surface is also due to the flattening of the strands' cross-sections. The roughness of the horizontal surfaces are also reduced by decreasing the strand-to-strand distance, which forces the extruded material to fill the intralayer cavities. On the other hand, the vertical surface roughness is weakly dependent on the strand-to-strand distance, as this parameter mostly affects the intra-layer bonds of the strands at the interior of the part, rather than the vertical external surfaces.

\section{Case 1}

$\mathrm{t} / \mathrm{D}=\mathbf{0 . 6}$

$\mathrm{s} / \mathrm{D}=\mathbf{1 . 4 0}$

(aligned)
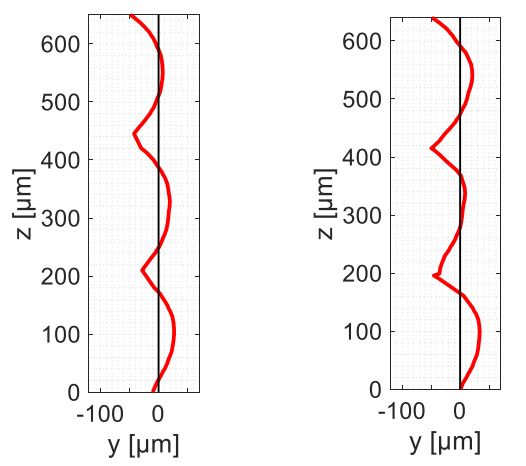

$-\mathrm{Ra}=18 \mu \mathrm{m}$
Case 3

$\mathrm{t} / \mathrm{D}=\mathbf{0 . 8}$

$\mathrm{S} / \mathrm{D}=\mathbf{1 . 0 0}$

(aligned)

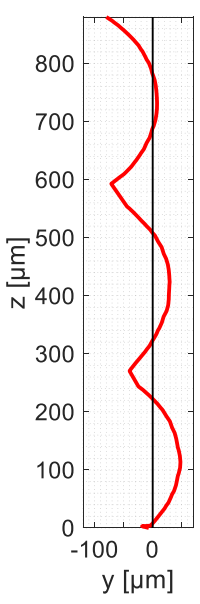

$-\mathrm{Ra}=25 \mu \mathrm{m}$
Case 4

$\mathrm{t} / \mathrm{D}=\mathbf{0 . 8}$

$\mathrm{s} / \mathrm{D}=1.20$

(aligned)

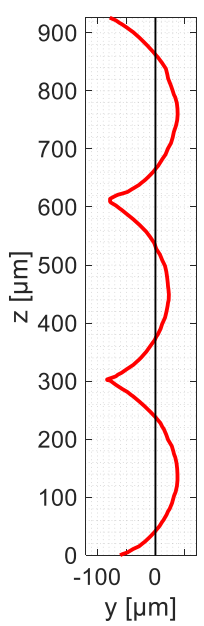

$-\mathrm{Ra}=26 \mu \mathrm{m}$

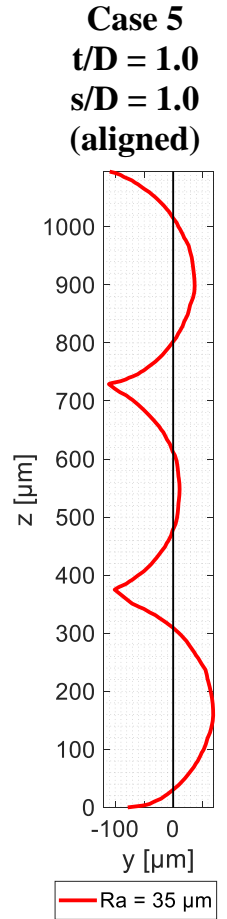

Case 6

$\mathbf{t} / \mathbf{D}=\mathbf{1 . 0}$

$\mathrm{s} / \mathrm{D}=\mathbf{1 . 1 5}$

(aligned)

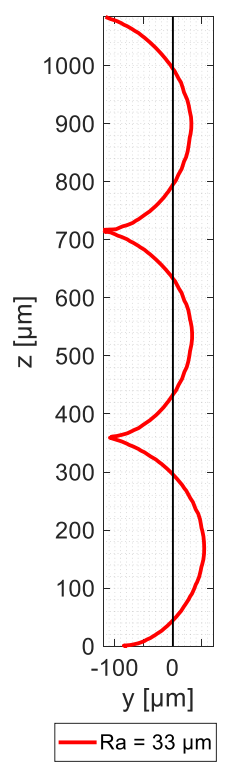

Figure 11. Vertical surface roughness of the mesostructures (aligned deposition configuration). 

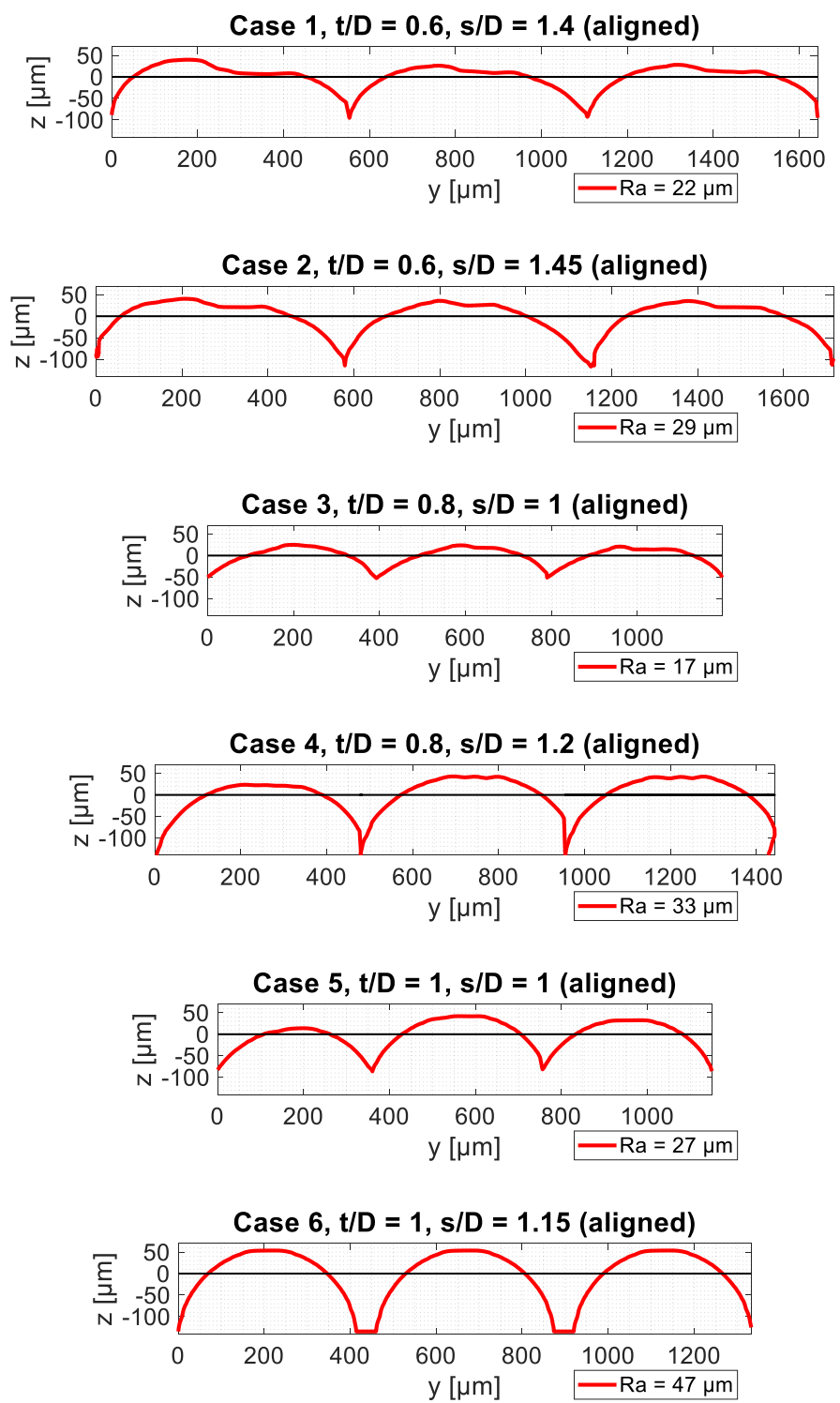

Figure 12. Horizontal surface roughness of the mesostructures (aligned deposition configuration).
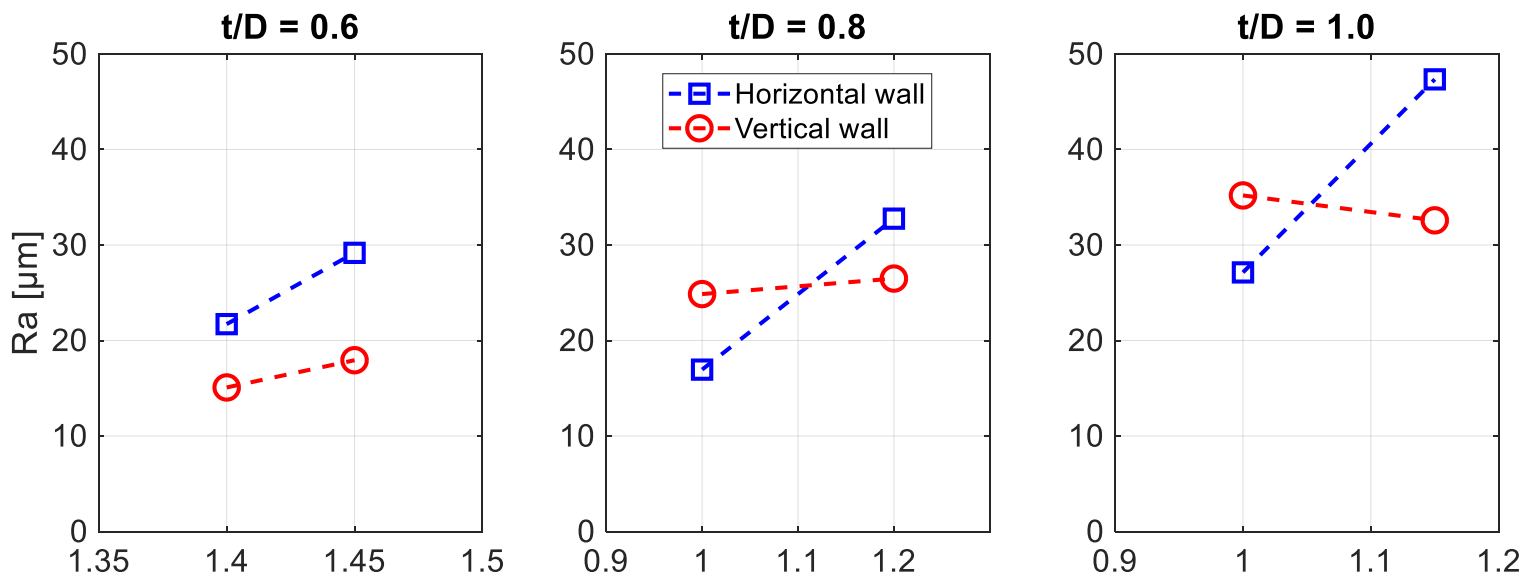

Strand-to-strand distance, s/D

Figure 13. Surface roughness of the simulated mesostructures with the aligned deposition configuration. 
This work presents a methodology using a computational fluid dynamics to simulate the mesostructure formation in material extrusion AM. The model takes into account the presence of the previously extruded material, which affects the deposition flow and the shape of subsequently extruded strands. The deposition of parallel strands in two different configurations, with aligned and skewed layers, was simulated. The influence of the layer thickness and the strand-to-stand distance on a representative volume element of the mesostructure was investigated. The simulated mesostructures agreed well with the experimental observations. It was found that both the layer thickness and the strand-to-stand distance influence the shapes of the strands and the formation of pores. The newly deposited strands can get significantly distorted by the presence of already deposited material, when the strandto-strand distance is smaller than the strand width. The distortions in the cross-sectional shape of the strands, which depend on the order of their deposition, affect the formation of the mesostructure. The numerical results were used to predict the influence of the processing parameters on the porosity, the inter- and intra-layer bond line densities, and the surface roughness. The simulations showed that decreasing the layer thickness and the strand-to-strand distance leads to smaller porosity and larger inter- and intralayer bond line densities. The advantage of the skewed configuration, i.e. a reduction of porosity, increases with the layer thickness. The surface roughness of the vertical and horizontal walls are improved by decreasing the layer thickness. Decreasing the strandto-strand distance also reduces the roughness of the horizontal surfaces. The quantitative predictions of the porosity, the bond line densities and the surface roughness open the possibility of performing numerical optimizations of the process parameters. Furthermore, the knowledge gained from the simulations can be used to create toolpath algorithms with more accurate geometrical representations of the strand profiles.

\section{Acknowledgments}

The authors would like to acknowledge the support of the Danish Council for Independent Research (DFF) | Technology and Production Sciences (FTP) (Contract No. 7017-00128).

\section{References}

[1] ISO/ASTM 52900:2015 Standard Terminology for Additive Manufacturing - General Principles - Terminology, ISO/ASTM Int. (2015) 1-9. doi:10.1520/F2792-12A.2.

[2] Wohlers Associates, Wohlers Report 2017, (2017).

[3] B.N. Turner, R. Strong, S.A. Gold, A review of melt extrusion additive manufacturing processes: I. Process design and modeling, Rapid Prototyp. J. 20 (2014) 192-204. doi:10.1108/RPJ-01-2013-0012.

[4] G.D. Goh, S. Agarwala, G.L. Goh, V. Dikshit, S.L. Sing, W.Y. Yeong, Additive manufacturing in unmanned aerial vehicles (UAVs): Challenges and potential, Aerosp. Sci. Technol. 63 (2017) 140-151. doi:10.1016/j.ast.2016.12.019.

[5] J.K. Placone, A.J. Engler, Recent Advances in Extrusion-Based 3D Printing for Biomedical Applications, Adv. Healthc. Mater. 7 (2018) 1-11. doi:10.1002/adhm.201701161.

[6] R.A. Buswell, W.R. Leal de Silva, S.Z. Jones, J. Dirrenberger, 3D printing using concrete extrusion: A roadmap for research, Cem. Concr. Res. 112 (2018) 37-49. doi:10.1016/j.cemconres.2018.05.006.

[7] B. Brenken, E. Barocio, A. Favaloro, V. Kunc, R.B. Pipes, Fused filament fabrication of fiber-reinforced polymers: A review, Addit. Manuf. 21 (2018) 1-16. doi:10.1016/j.addma.2018.01.002.

[8] A. Ghazanfari, W. Li, M.C. Leu, G.E. Hilmas, A novel freeform extrusion fabrication process for producing solid ceramic components with uniform layered radiation drying, Addit. Manuf. 15 (2017) 102-112. doi:10.1016/j.addma.2017.04.001.

[9] T.J. Hensen, T.G. Aguirre, C.L. Cramer, A.S. Wand, K. Ma, D.A. Prawel, J.D. Williams, T.B. Holland, Additive manufacturing of ceramic nanopowder by direct coagulation printing, Addit. Manuf. 23 (2018) 140-150. doi:10.1016/j.addma.2018.07.010.

[10] C. Gosselin, R. Duballet, P. Roux, N. Gaudillière, J. Dirrenberger, P. Morel, Large-scale 3D printing of ultra-high performance concrete - a new processing route for architects and builders, Mater. Des. 100 (2016) 102-109. doi:10.1016/j.matdes.2016.03.097.

[11] A.G. Tabriz, M.A. Hermida, N.R. Leslie, W. Shu, Three-dimensional bioprinting of complex cell laden alginate hydrogel structures, Biofabrication. 7 (2015). doi:10.1088/1758-5090/7/4/045012.

[12] M. Hospodiuk, M. Dey, D. Sosnoski, I.T. Ozbolat, The bioink: A comprehensive review on bioprintable materials, Biotechnol. Adv. 35 (2017) 217-239. doi:10.1016/j.biotechadv.2016.12.006. 
[13] S.H. Ahn, M. Montero, D. Odell, S. Roundy, P.K. Wright, Anisotropic material properties of fused deposition modeling ABS, Rapid Prototyp. J. 8 (2002) 248-257. doi:10.1108/13552540210441166.

[14] M. Dawoud, I. Taha, S.J. Ebeid, Mechanical behaviour of ABS: An experimental study using FDM and injection moulding techniques, J. Manuf. Process. 21 (2016) 39-45. doi:10.1016/j.jmapro.2015.11.002.

[15] C. Bellehumeur, L. Li, Modeling of Bond Formation Between Polymer Filaments in the Fused Deposition Modeling Process, J. Manuf. Process. 6 (2004) 170-178. doi:10.1016/S1526-6125(04)70071-7.

[16] C. Ziemian, S. Sharma, S. Ziemian, Anisotropic Mechanical Properties of ABS Parts Fabricated by Fused Deposition Modelling, in: D.M. Gokcek (Ed.), Mech. Eng., 2012. http://www.intechopen.com/books/mechanicalengineering/anisotropic- mechanical-properties-of-abs-parts-fabricated-by-fused-deposition-modeling.

[17] F.R. José, P.T. James, E.R. John, Mechanical behavior of acrylonitrile butadiene styrene fused deposition materials modeling, Rapid Prototyp. J. 9 (2003) 219-230. doi:10.1108/13552540310489604.

[18] C.S. Davis, K.E. Hillgartner, S.H. Han, J.E. Seppala, Mechanical strength of welding zones produced by polymer extrusion additive manufacturing, Addit. Manuf. 16 (2017) 162-166. doi:10.1016/j.addma.2017.06.006.

[19] H. Li, T. Wang, Z. Yu, The Quantitative Research of Interaction between Key Parameters and the Effects on Mechanical Property in FDM, Adv. Mater. Sci. Eng. 2017 (2017). doi:10.1155/2017/9152954.

[20] A.A. D'Amico, A. Debaie, A.M. Peterson, Effect of layer thickness on irreversible thermal expansion and interlayer strength in fused deposition modeling, Rapid Prototyp. J. 23 (2017) 943-953. doi:10.1108/RPJ-05-2016-0077.

[21] A.C. Abbott, G.P. Tandon, R.L. Bradford, H. Koerner, J.W. Baur, Process-structure-property effects on ABS bond strength in fused filament fabrication, Addit. Manuf. 19 (2018) 29-38. doi:10.1016/j.addma.2017.11.002.

[22] K.C. Ang, K.F. Leong, C.K. Chua, M. Chandrasekaran, Investigation of the mechanical properties and porosity relationships in fused deposition modelling-fabricated porous structures, Rapid Prototyp. J. 12 (2006) 100-105. doi:10.1108/13552540610652447.

[23] J.F. Rodriguez, J.P. Thomas, J.E. Renaud, Characterization of the mesostructure of fused-deposition acrylonitrilebutadiene-styrene materials, Rapid Prototyp. J. 6 (2000) 175-186. doi:https:// doi.org/10.1108/13552540010337056.

[24] A. Gleadall, I. Ashcroft, J. Segal, VOLCO: A predictive model for 3D printed microarchitecture, Addit. Manuf. 21 (2018) 605-618. doi:10.1016/j.addma.2018.04.004.

[25] J.M. Chacón, M.A. Caminero, E. García-Plaza, P.J. Núñez, Additive manufacturing of PLA structures using fused deposition modelling: Effect of process parameters on mechanical properties and their optimal selection, Mater. Des. 124 (2017) 143-157. doi:10.1016/j.matdes.2017.03.065.

[26] A. Bellini, S. Güçeri, Mechanical characterization of parts fabricated using fused deposition modeling, Rapid Prototyp. J. 9 (2003) 252-264. doi:10.1108/13552540310489631.

[27] C. Koch, L. Van Hulle, N. Rudolph, Investigation of mechanical anisotropy of the fused filament fabrication process via customized tool path generation, Addit. Manuf. 16 (2017) 138-145. doi:10.1016/j.addma.2017.06.003.

[28] K. Thrimurthulu, M.P. Pandey, N. V. Reddy, Optimum part deposition orientation in fused deposition modeling, Int. J. Mach. Tools Manuf. 44 (2004) 585-594. doi:https://doi.org/10.1016/j.ijmachtools.2003.12.004.

[29] D. Ahn, H. Kim, S. Lee, Surface roughness prediction using measured data and interpolation in layered manufacturing, J. Mater. Process. Technol. 209 (2009) 664-671. doi:10.1016/j.jmatprotec.2008.02.050.

[30] M. Taufik, P.K. Jain, A Study of Build Edge Profile for Prediction of Surface Roughness in Fused Deposition Modeling, J. Manuf. Sci. Eng. 138 (2016) 061002. doi:10.1115/1.4032193.

[31] A. Boschetto, L. Bottini, Accuracy prediction in fused deposition modeling, Int. J. Adv. Manuf. Technol. 73 (2014) $913-$ 928. doi:10.1007/s00170-014-5886-4.

[32] A. Boschetto, V. Giordano, F. Veniali, Modelling micro geometrical profiles in fused deposition process, Int. J. Adv. Manuf. Technol. 61 (2012) 945-956. doi:10.1007/s00170-011-3744-1.

[33] N. Mohan, P. Senthil, S. Vinodh, N. Jayanth, A review on composite materials and process parameters optimisation for the fused deposition modelling process, Virtual Phys. Prototyp. 12 (2017) 47-59. doi:10.1080/17452759.2016.1274490. 
O.A. Mohamed, S.H. Masood, J.L. Bhowmik, Optimization of fused deposition modeling process parameters: a review of current research and future prospects, Adv. Manuf. 3 (2015) 42-53. doi:10.1007/s40436-014-0097-7.

A.K. Sood, V. Chaturvedi, S. Datta, S.S. Mahapatra, Optimization of Process Parameters in Fused Deposition Modeling Using Weighted Principal Component Analysis, J. Adv. Manuf. Syst. 10 (2011) 241-259. doi:10.1142/S0219686711002181.

M. Jabbari, I. Baran, S. Mohanty, R. Comminal, M.R. Sonne, M.W. Nielsen, J. Spangenberg, J.H. Hattel, Multiphysics modelling of manufacturing processes: A review, Adv. Mech. Eng. 10 (2018) 1-31. doi:10.1177/1687814018766188.

J. Spangenberg, A. Uzal, M.W. Nielsen, J.H. Hattel, A robustness analysis of the bonding process of joints in wind turbine blades, Int. J. Adhes. Adhes. 85 (2018) 281-285. doi:10.1016/j.ijadhadh.2018.06.009.

N. Roussel, A. Gram, M. Cremonesi, L. Ferrara, K. Krenzer, V. Mechtcherine, S. Shyshko, J. Skocec, J. Spangenberg, O. Svec, L.N. Thrane, K. Vasilic, Numerical simulations of concrete flow: A benchmark comparison, Cem. Concr. Res. 79 (2016) 265-271. doi:10.1016/j.cemconres.2015.09.022.

E. Hovad, P. Larsen, J. Spangenberg, J.H. Walther, J. Thorborg, J.H. Hattel, Cavity prediction in sand mould production applying the DISAMATIC process, Powder Technol. 321 (2017) 204-217. doi:10.1016/j.powtec.2017.08.037.

J. Liu, K.L. Anderson, N. Sridhar, Direct Simulation of Polymer Fused Deposition Modeling (FDM) - An Implementation of the Multi-Phase Viscoelastic Solver in OpenFOAM, Int. J. Comput. Methods. 15 (2018) 1844002. doi:10.1142/S0219876218440024.

E. Bertevas, J. Férec, B.C. Khoo, G. Ausias, N. Phan-Thien, Smoothed particle hydrodynamics (SPH) modeling of fiber orientation in a 3D printing process, Phys. Fluids. 30 (2018) 103103. doi:10.1063/1.5047088.

R. Comminal, J.H. Hattel, J. Spangenberg, Numerical Simulations of Planar Extrusion and Fused Filament Fabrication of Non-Newtonian Fluids, Annu. Trans. Nord. Rheol. Soc. 25 (2017) 263-270.

J. Du, Z. Wei, X. Wang, J. Wang, Z. Chen, An improved fused deposition modeling process for forming large-size thinwalled parts, J. Mater. Process. Technol. 234 (2016) 332-341. doi:10.1016/j.jmatprotec.2016.04.005.

H. Xia, J. Lu, S. Dabiri, G. Tryggvason, Fully resolved numerical simulations of fused deposition modeling. Part I: fluid flow, Rapid Prototyp. J. 24 (2018) 463-476. doi:10.1108/RPJ-12-2016-0217.

H. Xia, J. Lu, G. Tryggvason, Fully Resolved Numerical Simulations of Fused Deposition Modeling. Part IISolidification, Residual Stresses, and Modeling of the Nozzle, Rapid Prototyp. J. 24 (2018) 973-987. doi:https:// doi.org/10.1108/RPJ-11-2017-0233.

R. Comminal, M.P. Serdeczny, D.B. Pedersen, J. Spangenberg, Numerical modeling of the strand deposition flow in extrusion-based additive manufacturing, Addit. Manuf. 20 (2018). doi:10.1016/j.addma.2017.12.013.

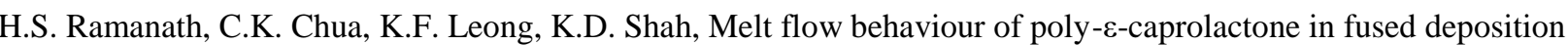
modelling, J. Mater. Sci. Mater. Med. 19 (2008) 2541-2550. doi:10.1007/s10856-007-3203-6.

A. Verma, P. Vishnoi, V. Sukhotskiy, E.P. Furlani, Numerical Simulation of Extrusion Additive Manufacturing : Fused Deposition Modeling, TechConnect Briefs. 4 (2018) 118-121.

M.P. Serdeczny, R. Comminal, D.B. Pedersen, J. Spangenberg, Experimental validation of a numerical model for the strand shape in material extrusion additive manufacturing, Addit. Manuf. 24 (2018). doi:10.1016/j.addma.2018.09.022.

R. Comminal, M.P. Serdeczny, D.B. Pedersen, J. Spangenberg, Numerical simulation of extrusion-based additive manufacturing - effect of the nozzle geometry on the strand cross-section, in: Euspen 's 18th Int. Conf., 2018: pp. 285286.

R. Comminal, M.P. Serdeczny, D.B. Pedersen, J. Spangenberg, Numerical modeling of the material deposition and contouring precision in fused deposition modeling, Proc. 29th Solid Free. Fabr. Symp. Austin, TX. (2018) 1855-1864. http://sffsymposium.engr.utexas.edu/TOC2018.

M.P. Serdeczny, R. Comminal, D.B. Pedersen, J. Spangenberg, Numerical Study of the Impact of Shear Thinning Behaviour on the Strand Deposition Flow in the Extrusion-Based Additive Manufacturing, in: Euspen's 18th Int. Conf. Exhib. Venice, IT, 2018: pp. 283-284.

C.W. Hirt, B.D. Nichols, Volume of fluid (VOF) method for the dynamics of free boundaries, J. Comput. Phys. 39 (1981) 
2 [54] M. Sussman, E.G. Puckett, A Coupled Level Set and Volume-of-Fluid Method for Computing 3D and Axisymmetric 3 Incompressible Two-Phase Flows, J. Comput. Phys. 162 (2000) 301-337. doi:10.1006/jcph.2000.6537.

4 [55] R. Comminal, J. Spangenberg, J.H. Hattel, Cellwise conservative unsplit advection for the volume of fluid method, J. Comput. Phys. 283 (2015) 582-608. doi:10.1016/j.jcp.2014.12.003. 